\title{
RESEARCH
}

Open Access

\section{Effect of extracorporeal shock wave therapy on different severities of carpal tunnel syndrome}

\author{
Usama Mohammad Rashad', Nirmeen A. Kishk², Waleed Talat Mansour', Amani M. Nawito ${ }^{3}$, \\ Alshaimaa Sobhi Khali ${ }^{3^{*}}$ (D) Hanan Helmy ${ }^{2}$ and Tarek Kamal Zayed ${ }^{4}$
}

\begin{abstract}
Background: Carpal tunnel syndrome (CTS) is the most common peripheral nerve entrapment syndrome worldwide.

Objective: The current work aimed at determining the effect of extracorporeal shock wave therapy (ESWT) on different severities of carpal tunnel syndrome.

Subjects and methods: Sixty patients with unilateral carpal tunnel syndrome were participated in this study. They were divided into three equal groups according to severity (mild, moderate, and severe). All patients received shock wave therapy one session per week, for 6 weeks. Nerve conduction studies (NCs) were done for the median nerve. Pinch dynamometer was used to measure the degree of lateral pinch power, and Visual Analogue Scale (VAS) was used to assess degree of pain and Ultrasonography analysis measured cross-sectional area (CSA) of median nerve for all patients at the beginning and at the end of the study.

Results: There was significant improvement $(P<0.05)$ in sensory conduction parameters, CSA, lateral pinch power, and VAS after shock wave therapy in all groups. Also, there was significant improvement in motor latency, motor, and sensory amplitudes after shock wave therapy in mild and moderate groups only $(P<0.05)$. In all group samples, results revealed a highly significant correlations $(p<0.001)$ between peak sensory latency (PSL) with VAS, CSA with motor latency (ML), CSA with PSL, CSA with VAS, CSA with lateral pinch, and ML with lateral pinch.
\end{abstract}

Conclusion: It is recommended to use ESWT as a conservative treatment in patients with different severities of CTS, except patients with severe motor affection.

Trial registration: PACTR201804003293116. Registered on 26 April 2018 as retrospective registration in the pan African clinical trial registry (www.pactr.org).

Keywords: Carpal tunnel syndrome, Ultrasound, Extracorporeal shock wave therapy

\section{Introduction}

Carpal tunnel syndrome (CTS) is considered the most common entrapment neuropathy resulting from peripheral nerve trapping, and its prevalence in adult's general population has been reported $2.7-5.8 \%$; it has been

\footnotetext{
* Correspondence: alshaimaa.sobhi@kasralainy.edu.eg

${ }^{3}$ Clinical Neurophysiology Unit, Faculty of Medicine, Cairo University, 71 Kasr Alainy street, Cairo 11562, Egypt

Full list of author information is available at the end of the article
}

reported that $50 \%$ patients have bilateral CTS [1]. The median nerve is commonly compressed where it passes in the carpal tunnel which is the most common form of entrapment neuropathies [2]. It typically presents with numbness, tingling, nocturnal pain, and at times, weakness in the hand [3].

The risk factors for CTS include repetitive wrist stress, obesity, and pregnancy, whereas secondary causes include lesions within the carpal tunnel, metabolic causes,
Springer Open

(c) The Author(s). 2020 Open Access This article is licensed under a Creative Commons Attribution 4.0 International License, which permits use, sharing, adaptation, distribution and reproduction in any medium or format, as long as you give appropriate credit to the original author(s) and the source, provide a link to the Creative Commons licence, and indicate if changes were made. The images or other third party material in this article are included in the article's Creative Commons licence, unless indicated otherwise in a credit line to the material. If material is not included in the article's Creative Commons licence and your intended use is not permitted by statutory regulation or exceeds the permitted use, you will need to obtain permission directly from the copyright holder. To view a copy of this licence, visit http://creativecommons.org/licenses/by/4.0/. 
and infection. Unlike other progressive diseases, CTS is characterized by remission and recurrence; hence, its prognosis is often uncertain. Although there are many conservative forms of management, such as wrist splint, steroid injections, and therapeutic ultrasound, their effectiveness is typically insignificant or short-lived [4].

Proper diagnosis of CTS generally involves the evaluation of clinical signs and symptoms in combination with neurophysiological tests as motor and sensory nerve conduction studies in addition to high-resolution ultrasonography that has been used as a useful method for the diagnosis of CTS. The measurement of crosssectional area (CSA) of the median nerve at the wrist is the most widely used ultrasonography method in CTS diagnosis [5].

A shock wave is a sonic pulse with certain physical properties. There is a high peak pressure, a fast initial rise in pressure during a period of less than $10 \mathrm{~ns}$, a low tensile amplitude, a short life cycle, and a broad frequency spectrum [6].

ESWT is a noninvasive technique which uses singlepulse acoustic waves. These pulses are generated outside the body and focused on a specific part of the body [7]. It relieves the pain of peripheral nerve damage and enhances local arterial remodeling and cellular regeneration [8].

Extracorporeal shockwave therapy (ESWT) has been used in the treatment of several painful inflammatory soft tissue conditions with an acceptable successful outcome [9].

This study aimed to assess the effect of ESWT on different severities of carpal tunnel syndrome.

\section{Subjects and methods}

This is a case-control experimental study, conducted on 60 patients with unilateral carpel tunnel syndrome recruited from Al Sahel Teaching Hospital and Neurology outpatient clinic of Cairo University Hospitals in the period from November 2016 to July 2017.

\section{Subjects}

Patients were from both genders, and their ages ranged from 20 to 40 years old and height ranged from 155 to $185 \mathrm{~cm}$. They were subdivided into three groups with each twenty patients: group A; mild CTS (16 females, 4 males) with their mean age $(33.40+4.35)$ years and height $(164.70+7.49) \mathrm{cm}$, group B; moderate CTS $(3$ males, 17 females), mean age $(33.40+3.70)$ years and height $(164.55+5.94) \mathrm{cm}$, and group C; severe CTS $(4$ males, 16 females), mean age $(33.60+4.48)$ years and height $(164.45+5.47) \mathrm{cm}$. Duration of symptoms was more than 3 months. Patients with the following were included: discomfort pain in thenar, positive Phalen's maneuver, Tinel's sign, or carpal compression test.
Patients were assigned into three equal groups according to the proposed scheme of the American Association of Neuromuscular and Electrodiagnostic Medicine [10]:

Mild CTS: prolonged (relative or absolute) sensory or mixed nerve action potential distal latency (orthodromic, antidromic, or palmar) \pm sensory nerve action potential (SNAP) amplitude below the lower limit of normal.

Moderate CTS: abnormal median sensory latencies as above and (relative or absolute) prolongation of median motor distal latency.

Severe CTS: prolonged median motor and sensory distal latencies, with either an absent SNAP or mixed nerve action potential, or low amplitude or absent thenar compound muscle action potential (CMAP). Needle examination often reveals fibrillations, reduced recruitment, and motor unit potential changes.

Patients with the following criteria were excluded from the study: juvenile diabetes, orthopedic deformities in the upper limb, vascular disease at the wrist and/or the hand, cortisone therapy up to 6 weeks, cognitive or psychiatric disorders, body mass index (BMI) $>30 \mathrm{~kg} / \mathrm{m} 2$, pregnancy, cervical spondylosis, osteoporosis, or hypothyroidism.

\section{Methods}

After selection of the participants, the purpose of the study was adequately explained to each participant.

Informed written consent was obtained from all individual participants included in the study.

All patients were submitted to clinical examination, median nerve NCS, and ultrasound (US) were done prior to and post-application of ESWT.

\section{Assessment techniques \\ Neurophysiological study of the median nerve}

This was done using Dantec key point, USA, for measuring nerve conduction velocity (NCV), motor latency, motor amplitude, peak sensory latency, sensory amplitude, and sensory conduction velocity of median nerve pre and post-treatment program for three groups. The normal reference values are distal latency $\leq 4.4 \mathrm{~ms}$, conduction velocity $\geq 50.0 \mathrm{~m} / \mathrm{s}$, and the amplitude $\geq 4 \mathrm{mV}$ from baseline to peak [11].

\section{Ultrasonography analysis of median nerve}

This took place in the neurosonology unit at Neurology department, Kasr-alainy School of medicine, Cairo University and was performed by a single experienced certified neurosonographer (European Society of Neuro-sonology and Cerebral Hemodynamics "ENSCH"), who was blinded to the subjects' clinical data throughout the entire study. The studies were carried out using a high-resolution ultrasonography 
instrument (PHILIPS IU22 xMATRIX, California, US, L 13-17MHZ linear transducer).

All patients were evaluated in the resting neutral position with the palm up. The full course of the median nerve in the carpal tunnel was assessed in both the transverse and sagittal planes to assess the CSA.

Transverse image captured using a High frequency (13-17 MHz) Phased array transducer. Probe oriented to the left side of the screen and transverse image at the carpal tunnel inlet detected anatomically by distal wrist crease; yet, measurements were taken only when the scaphoid tubercle and the pisiform bone at the proximal margin of flexor retinaculum were seen. The median nerve CSA and the transverse (major axis) and anteroposterior (minor axis) diameters were measured in the transverse plane at the proximal boundary of the carpal tunnel at the point of posterior angulation of the median nerve. The CSA was measured 3 times, and the average value was used for analysis. The cross-sectional area of the median nerve was calculated by using a continuous boundary trace just within the echogenic boundary of the nerve $[12,13]$.

Pinch dynamometer (Hydraulic Pinch gauge, Saehan, SH5005, Korea) was used to measure the degree of lateral pinch power pre and post-treatment program for all participants in the study.

\section{Visual Analogue Scale (VAS)}

It was used to assess the pain level pre- and posttreatment program for all patients in three groups [14].

\section{Treatment method}

\section{Shock wave unit}

The device Chattanooga, Intelect RPW (USA) was calibrated by the manufacturer routinely, and it was calibrated before starting the study, to be used as treatment.

The treatment protocol was given for three groups one session per week for 6 weeks. Each patient received shock wave therapy for 2000 shocks at energy flux density (EFD) of $0.03 \mathrm{~mJ} / \mathrm{mm}^{2}$ and $1.6 \mathrm{bar}$ in the area between the thenar and hypothenar eminences [7]. The probe was oriented perpendicular to the patient's palm.

\section{Statistical analysis}

Data was statistically described in terms of mean \pm standard deviation (SD), median and range ,and percentages when appropriate. Paired $t$ test was used to detect the difference between pre and post-treatment within each group. Correlation between various variables was done. $P$ values less than 0.05 was considered statistically significant. All statistical calculations were done using the SPSS software for medical statistics version 20 .

\section{Results}

Table 1 showed the participant's demographic, clinical, neurophysiological, and neurosonological results of the affected side at the onset and follow-up.

Comparative data between pre-and post-treatment lateral pinch showed that there were highly significant differences within the mild group $(P=0.0001)$, moderate group $(P=0.0001)$, and severe group $(P=0.005)$ as shown in Table 2.

Comparison between pre-and post-treatment neurophysiological data (motor latency, motor amplitude, sensory peak latency, and sensory conduction velocity) is shown in Table 3 . There were highly significant differences between pre- and post-motor amplitude, peak sensory latency, sensory amplitude, and sensory conduction velocity of median nerve within the mild group $(P=$ 0.0001, $P=0.0001, P=0.0001, P=0.002$; respectively) and moderate group $(P=0.0001, P=0.002, P=0.005, P$ $=0.001$; respectively). The severe group showed significant differences between pre- and post-sensory latency and sensory conduction velocity of median nerve only ( $P$ $=0.003, P=0.001$; respectively).

The comparison between the pre-and post-treatment mean value of cross-sectional area of the median nerve showed that there were highly significant differences within the mild group $(P=0.0001)$, moderate group ( $P$ $=0.0001) \mathrm{m}$ and severe group $(P=0.001)$ as shown in Table 4.

Correlation between different variables post-treatment in all group sample results revealed the following:

Highly significant $(P<0.05)$ negative correlations were observed between motor latency of median nerve with lateral pinch $(r=-0.54)$ and between cross-section area of median nerve with lateral pinch $(r=-0.52)$.

A highly significant $(P<0.05)$ positive correlation between peak sensory latency of median nerve and VAS $(r=0.67)$.

Highly significant $(P<0.05)$ positive correlations between CSA of median nerve with motor latency $(r$

Table 1 Demographic data

\begin{tabular}{lll}
\hline Demographic and clinical data & Patients $(N=60)$ \\
\hline Sex & Females & $49(81.7 \%)$ \\
Age & Males & $11(18.3 \%)$ \\
& Range & $20-40$ years \\
Height & median & 34 years \\
& Range & $155-185$ \\
Affected hand & median & 170 \\
& Right & $40(66.7 \%)$ \\
\hline
\end{tabular}

$N$ number, $y$ year 
Table 2 Comparison between pre- and post-clinical data within mild, moderate, and severe groups

\begin{tabular}{lllll}
\hline \multicolumn{2}{l}{ Clinical assessment } & & Mean \pm SD & $P$ value \\
\hline Lateral pinch & Mild & Pre & $14.50 \pm 3.14$ & $\mathbf{0 . 0 0 0 1}^{\text {b }}$ \\
& & Post & $17.01 \pm 4.02$ & \\
& Moderate & Pre & $12.94 \pm 3.26$ & $\mathbf{0 . 0 0 0 1}^{\text {b }}$ \\
& & Post & $14.98 \pm 3.87$ & \\
& Severe & Pre & $10.26 \pm 3.02$ & $\mathbf{0 . 0 0 5}^{\text {a }}$ \\
& & Post & $11.45 \pm 3.41$ & \\
VAS & Pild & Pre & $7.65 \pm 0.48$ & $\mathbf{0 . 0 0 0 1}^{\text {b }}$ \\
& & Post & $5.20 \pm 1.00$ & \\
& Moderate & Pre & $8.15 \pm 0.58$ & $\mathbf{0 . 0 0 0 1}^{\text {b }}$ \\
& & Post & $5.80 \pm 1.00$ & \\
& Severe & Pre & $8.25 \pm 0.68$ & $\mathbf{0 . 0 0 1}^{\text {a }}$ \\
& & Post & $7.40 \pm 1.31$ & \\
\hline
\end{tabular}

VA Visual Analogue Scale, SD standard deviation

${ }^{a} p$ value $<0.05$ is considered significant

${ }^{\mathrm{b}} p$ value $<0.001$ is considered highly significant

$=0.94)$, peak sensory latency $(r=0.63)$, and VAS $(r$ $=0.69$ ).

\section{Discussion}

This study was mainly concerned with the effect of ESWT on different severities of carpal tunnel syndrome.

Results of this study showed a significant improvement in VAS and lateral pinch power of the affected hand after ESWT in all groups.

Paoloni and colleagues [15] study the effect of US and ESWT on mild and moderate CTS, and they concluded that patients in ESWT group show greater results regarding pain at 12 weeks follow-up when compared with the US group $(p<0.05)$, and benefits persist 3 months after the end of treatment. Also, it is reported that after three sessions of ESWT alone, patients with mild-tomoderate CTS showed pain relief and better functionality, as compared to ultrasound and cryo-ultrasound therapy.

In addition, WU and colleagues [4] suggested that a significant improvement in the Visual Analogue Scale (VAS) and the Boston Carpal Tunnel Syndrome Questionnaire (BCTQ) scores were found in the patients' group when compared to the control group.

The results of the present study come in line with Vahdatpour and colleagues [1], who evaluated the effect of extracorporeal shock wave therapy on the treatment of patients with CTS. This improvement was found in clinical symptoms, so they recommended to use ESWT for treatment in patients with CTS.

The results of the present study agreed with Romeo and colleagues [9], who stated that the ESWT resulted in significant improvements of symptoms in patients
Table 3 comparison between pre- and post-neurophysiological parameters within mild, moderate, and severe groups

\begin{tabular}{|c|c|c|c|c|}
\hline \multicolumn{3}{|c|}{ Neurophysiological parameters } & \multirow{2}{*}{$\frac{\text { Mean } \pm \text { SD }}{4.20 \pm 0.14}$} & \multirow{2}{*}{$\frac{P \text { value }}{0.0001^{b}}$} \\
\hline Motor latency & mild & Pre & & \\
\hline & & Post & $3.78 \pm 0.18$ & \\
\hline & moderate & Pre & $5.99 \pm 0.37$ & $0.0001^{b}$ \\
\hline & & Post & $5.50 \pm 0.49$ & \\
\hline & Severe & Pre & $8.23 \pm 0.46$ & 0.080 \\
\hline & & Post & $8.10 \pm 0.52$ & \\
\hline \multirow[t]{6}{*}{ Motor amplitude } & Mild & Pre & $4.51 \pm 0.27$ & $0.0001^{\mathrm{b}}$ \\
\hline & & Post & $5.24 \pm 0.58$ & \\
\hline & Moderate & Pre & $4.27 \pm 0.19$ & $0.0001^{b}$ \\
\hline & & Post & $4.84 \pm 0.31$ & \\
\hline & Severe & Pre & $1.43 \pm 0.45$ & 0.162 \\
\hline & & Post & $1.59 \pm 0.65$ & \\
\hline \multirow[t]{6}{*}{ Sensory peak latency } & Mild & Pre & $4.34 \pm 0.19$ & $0.0001^{b}$ \\
\hline & & Post & $3.79 \pm 0.28$ & \\
\hline & moderate & Pre & $5.70 \pm 0.53$ & $0.002^{\mathrm{a}}$ \\
\hline & & Post & $5.19 \pm 0.64$ & \\
\hline & Severe & Pre & $9.19 \pm 2.27$ & $0.003^{a}$ \\
\hline & & Post & $8.55 \pm 2.13$ & \\
\hline \multirow[t]{6}{*}{ Sensory amplitude } & Mild & Pre & $18.45 \pm 1.30$ & $0.0001^{b}$ \\
\hline & & Post & $21.38 \pm 2.14$ & \\
\hline & Moderate & Pre & $12.74 \pm 1.20$ & $0.005^{\mathrm{a}}$ \\
\hline & & Post & $14.49 \pm 2.53$ & \\
\hline & Severe & Pre & $2.76 \pm 1.34$ & 0.170 \\
\hline & & Post & $3.13 \pm 1.87$ & \\
\hline \multirow[t]{6}{*}{ Sensory CV } & Mild & Pre & $41.16 \pm 3.85$ & $0.002^{\mathrm{a}}$ \\
\hline & & Post & $45.94 \pm 4.27$ & \\
\hline & Moderate & Pre & $34.62 \pm 2.15$ & $0.004^{\mathrm{a}}$ \\
\hline & & Post & $37.27 \pm 3.31$ & \\
\hline & Severe & Pre & $23.53 \pm 10.33$ & $0.006^{\mathrm{a}}$ \\
\hline & & Post & $25.14 \pm 11.17$ & \\
\hline
\end{tabular}

$S D$ standard deviation, $C V$ conduction velocity ${ }^{\mathrm{a}} p$ value $<0.05$ is considered significant

${ }^{\mathrm{b}} p$ value $<0.001$ is considered highly significant

with pillar pain after carpal tunnel release surgery, and there was a significant improvement with the mean VAS score decreased from 6.18 to 0.44 ( \pm 0.63$) 120$ days after treatment.

In addition, Notarnicola and colleagues [16] compared the effect of ESWT and nutraceutical composed of Echinacea angustifolia, alpha lipoic acid, conjugated linoleic acid, and quercetin (perinerv) in patients with CTS. Results showed that shock wave therapy is an effective treatment for reducing symptoms and improvement in quality of life of patients. 
Table 4 Comparison between pre- and post-cross-section area of median nerve within mild, moderate, and severe groups

\begin{tabular}{|c|c|c|c|c|c|c|}
\hline & \multicolumn{6}{|c|}{ Cross-section area of median nerve $\left(\mathrm{mm}^{2}\right)$} \\
\hline & \multicolumn{2}{|c|}{ Mild group $(n=20)$} & \multicolumn{2}{|c|}{ Moderate group $(n=20)$} & \multicolumn{2}{|c|}{ Severe group $(n=20)$} \\
\hline & Pre & Post & Pre & Post & Pre & Post \\
\hline Mean $( \pm S D)$ & $8.23 \pm 0.51$ & $7.08 \pm 0.45$ & $9.46 \pm 0.65$ & $8.38 \pm 0.65$ & $13.32 \pm 1.93$ & $12.09 \pm 1.69$ \\
\hline$P$ value & $0.0001^{a}$ & & $0.0001^{a}$ & & $0.0001^{a}$ & \\
\hline
\end{tabular}

$S D$ standard deviation, $\mathrm{mm}$ millimeter, $n$ number

${ }^{a} p$ value $<0.001$ is considered highly significant

Results of the present study revealed that an improvement in electrophysiological findings (motor latency, peak sensory latency, motor and sensory amplitudes, and sensory nerve conduction velocity) of median nerve after ESWT in mild and moderate groups while in severe group results revealed that an improvement in peak sensory latency and sensory nerve conduction velocity only after ESWT.

Vahdatpour and colleagues [1] agreed with the results of the present study; they found that an improvement in the electrophysiological findings of median nerve in the patients with carpal tunnel following treatment with ESWT.

Also, Hausner and colleagues [17] concluded that lowenergy ESWT could induce marked recovery of nerve regeneration, nerve conduction velocity $(\mathrm{NCV})$, and amplitude in rats treated with nerve autografts of the sciatic nerve.

Results of the present study revealed an improvement in ultrasonography finding (cross-section area) of median nerve after ESWT in all groups.

In agreement with the results of the current study, the results of WU and colleagues [9] suggested that a significant improvement in CSA of the median nerve was observed in the patients group compared to the control one.

Moreover, WU and colleagues [9] found a significant decrease in the CSA of the median nerve after rESWT as compared to the control group. They concluded that ESWT is an effective method for relieving symptoms of CTS as corticosteroid injection. In addition to that, rESWT is a noninvasive method in contrast to corticosteroid injection.

Also, Ke and colleagues [18] suggested that ESWT is a valuable method for treatment of mild to moderate CTS, and that its effect is more detectable in moderate cases than mild ones because mild CTS partially resolves spontaneously. In addition, the effect of single-session ESWT is insignificant as it is a cumulative process.

Results of the present study revealed a highly significant correlations between electrophysiological and clinical variables after ESWT in all group samples. In addition, highly significant correlations were observed among CSA with clinical symptoms (lateral pinch and VAS) and CSA with nerve conduction studies. Hence, CSA of the median nerve can be used as a screening tool for detection as well as discrimination of different severities of CTS. Also, VAS and lateral pinch can be used for detection and assessment of the efficacy of treatment in CTS.

In agreement with the results of the current study, the results of Hala RE and colleagues [19] found a significant correlation between the CSAs of the median nerve in the wrist and median nerve conduction studies. Correlating the CSA of the median nerve in the wrist with the NCS results showed significant positive correlations with both motor latency and sensory latency in addition to a significant negative correlation with both motor amplitude and sensory amplitude. The high-resolution ultrasonography showed $96.1 \%$ accuracy in the detection of CTS.

On the other hand, the findings of the present study disagree with Wu and Lun [20], who suggested that ESWT may be useless to peripheral nerve injuries and can be used in the treatment of musculoskeletal disorders only.

Results of the present study could be explained as ESWT stimulates the production of endothelial nitric oxide [21], angiogenesis [22], and neurogenesis [23] via the involvement of the vascular endothelial growth factor resulting in the decrease of the local inflammation and release of the pressure on the median nerve.

Moreover, ESWT produces anti-inflammatory effects through reduction of the levels of calcitonin gene-related peptide [24]. Low energy flux density (EFD) levels of shockwaves induce a significant decreasing in proinflammatory substances such as nuclear factor-kappa B (NF-kB) [9]. ESWT may also reduce inflammation of the soft tissues around the median nerve, which results in reduced pressure on the median nerve.

\section{Conclusion}

Extracorporeal shock wave therapy is an effective method for treating patients with different severities of carpal tunnel syndrome, except patients with severe motor affection.

\section{Limitations}

Unavailable information of normative data regarding lateral pinch test among Egyptian patients so further study of the sensitivity and specificity of our results could not be assessed. 


\section{Abbreviations}

BCTQ: Boston Carpal Tunnel Syndrome Questionnaire; BMl: Body mass index; CMAP: Compound muscle action potential; CSA: Cross-sectional area; CTS: Carpal tunnel syndrome; EFD: Energy flux density; ESWT: Extracorporeal shock wave therapy; ML: Motor latency; mm: Millimeter; ms: Millisecond; N: Number; NCs: Nerve conduction studies; NCV: Nerve conduction velocity; PSL: Peak sensory latency; SD: Standard deviation; SNAP: Sensory nerve action potential; US: Ultrasound; VAS: Visual Analogue Scale; Y: Year

\section{Acknowledgements}

Not applicable

\section{Authors' contributions}

UMR and NAK designed the analyses. NAK, WTM, AMN, and TKZ collected and analyzed the data. ASK and TKZ wrote the first draft of the manuscript. UMR, NAK, WTM, AMN, ASK, HH, and TKZ critically reviewed the manuscript. All authors read and approved the final manuscript.

\section{Funding}

This research did not receive any specific grant from funding agencies in the public, commercial, or not-for-profit sectors.

\section{Availability of data and materials}

The data that support the findings of this study are available on request from the corresponding author [ASK]. The data are not publicly available due to data protection and the fact they contain information that could compromise research participant privacy/consent.

\section{Ethics approval and consent to participate}

All procedures performed in studies involving human participants were in accordance with the ethical standards of the institutional and/or national research committee and with the 1964 Helsinki declaration and its later amendments or comparable ethical standards.

This article does not contain any studies with animals performed by any of the authors.

The protocol of the study was discussed and approved by the Research Ethical Committee-Faculty of Physical Therapy in 5 July 2015, then approved by Cairo University at the end of July 2015 with Reference Number: P.T.REC/ 012/00975.

\section{Consent for publication}

Not applicable

\section{Competing interests}

The authors declare that they have no competing interests.

\section{Author details}

'Department of Physical Therapy for Neuromuscular Disorder and Its Surgery, Faculty of Physical Therapy, Cairo University, Cairo, Egypt. ${ }^{2}$ Department of Neurology, Faculty of Medicine, Cairo University, Cairo, Egypt. ${ }^{3}$ Clinical Neurophysiology Unit, Faculty of Medicine, Cairo University, 71 Kasr Alainy street, Cairo 11562, Egypt. ${ }^{4}$ Department of Physical Therapy, El Sahel Teaching Hospital, General Organization of Hospitals and Institutes, Cairo, Egypt.

Received: 28 March 2019 Accepted: 13 May 2020

Published online: 27 May 2020

\section{References}

1. Vahdatpour B, Kiyani A, Dehghan F. Effect of extracorporeal shock wave therapy on the treatment of patients with carpal tunnel syndrome. Adv Biomed Res. 2016;5:120.

2. Alrawashdeh O. Prevalence of Asymptomatic Neurophysiological carpal tunnel syndrome in 130 healthy individuals. Neurol Int. 2016;8(4):6553.

3. Lozano-Calderón S, Anthony S, Ring D. The quality and strength of evidence for etiology: example of carpal tunnel syndrome. J Hand Surg Am. 2008:33(4):525-38.

4. Wu YT, Ke MJ, Chou YC, Chang CY, Lin CY, Li TY, et al. Effect of radial shock wave therapy for carpal tunnel syndrome: a prospective randomized, doubleblind, placebo-controlled trial. J Orthop Res. 2016;34(6):977-84.
5. Visser LH, Smidt MH, Lee ML. High resolution sonography versus EMG in the diagnosis of carpal tunnel syndrome. J Neurol Neurosurg Psychiatry. 2008; 79(1):63-7.

6. Schleberger R, Delius M, Dahmen GP, et al. Orthopedic extracorporeal shock wave therapy (ESWT). Method analysis and suggestion of a prospective study design - consensus report, in High Energy Shock Waves in Medicine. 1997; pp. 108-111. Eds., Chaussy, C., Eisenberger, F., Jocham, D., Wilbert, D., Stuttgart. George Thieme Verlag. .

7. Seok H, Kim SH. The effectiveness of extracorporeal shock wave therapy vs. local steroid injection for management of carpal tunnel syndrome: a randomized controlled trial. Am J Phys Med Rehabil. 2013:92(4):327-34.

8. Lee $\mathrm{JH}, \mathrm{Cho} \mathrm{SH}$. Effect of extracorporeal shock wave therapy on denervation atrophy and function caused by sciatic nerve injury. J Phys Ther Sci. 2013; 25(9):1067-9.

9. Romeo P, D'Agostino C, Lazzerini A, Sansone VC. Extracorporeal shock wave therapy in pillar pain after carpal tunnel release: a preliminary study. Ultrasound Med Biol. 2011:37(10):1603-8.

10. Stevens JC. AAEM minimonograph \#26: the electrodiagnosis of carpal tunnel syndrome. American Association of Electrodiagnostic Medicine Muscle Nerve. 1997:20(12):1477-86.

11. Cranford CS, Ho JY, Kalainov DM, Hartigan BJ. Carpal tunnel syndrome. J Am Acad Orthop Surg. 2007:15(9):537-48.

12. Buchberger W, Schön G, Strasser K, Jungwirth W. High-resolution ultrasonography of the carpal tunnel. J Ultrasound Med. 1991;10(10):531-7.

13. Buchberger W, Judmaier W, Birbamer G, Lener M, Schmidauer C. Carpal tunnel syndrome: diagnosis with high-resolution sonography. AJR Am J Roentgenol. 1992;159(4):793-8.

14. Wewers ME, Lowe NK. A critical review of visual analogue scales in the measurement of clinical phenomena. Res Nurs Health. 1990 Aug;13(4):22736.

15. Paoloni M, Tavernese E, Cacchio A, D'Orazi V., loppolo F., Fini M., et al. Extracorporeal shock wave therapy and ultrasound therapy improve pain and function in patients with carpal tunnel syndrome. A randomized controlled trial. Eur J Phys Rehabil Med. 2015; 51(5):521-528.

16. Notarnicola A, Maccagnano G, Tafuri S, Fiore A, Pesce V, Moretti B. Comparison of shock wave therapy and nutraceutical composed of Echinacea angustifolia, alpha lipoic acid, conjugated linoleic acid and quercetin (perinerv) in patients with carpal tunnel syndrome. Int J Immunopathol Pharmacol. 2015;28:256-62.

17. Hausner T, Pajer K, Halat G, Hopf R, Schmidhammer R, Redl H, et al. Improved rate of peripheral nerve regeneration induced by extracorporeal shock wave treatment in the rat. Exp Neurol. 2012;236:363-70.

18. Ke MJ, Chen LC, Chou YC, Li TY, Chu HY, Tsai CK, et al. The dose-dependent efficiency of radial shock wave therapy for patients with carpal tunnel syndrome: a prospective, randomized, single-blind, placebo-controlled trial. Sci Rep. 2016;6.

19. El Habashy HR, El Hadidy RA, Ahmed SM, El Sayed BB, Ahmed AS. Carpal tunnel syndrome grading using high-resolution ultrasonography. J Clin Neurophysiol. 2017;34(4):353-8.

20. Wu YH, Lun JJ, Chen WS and Chong FC. The electrophysiological and functional effect of shock wave on peripheral nerves. Conf Proc IEEE Eng Med Biol Soc. 2007; 2007:2369-2372.

21. Mariotto S, Cavalieri E, Amelio E. Extracorporeal shock waves: from lithotripsy to anti-inflammatory action by NO production. Nitric Oxide. 2005; 12:89-96.

22. Ito K, Fukumoto $Y$, Shimokawa H. Extracorporeal shock wave therapy as a new and non-invasive angiogenic strategy. Tohoku J Exp Med. 2009;219:1-9.

23. Jin K, Mao XO, Greenberg DA. Vascular endothelial growth factor stimulates neurite outgrowth from cerebral cortical neurons via Rho kinase signaling. J Neurobiol. 2006;66:236-42

24. Takahashi N, Wada Y, Ohtori S. Application of shock waves to rat skin decreases calcitonin gene-related peptide immunoreactivity in dorsal root ganglion neurons. Auton Neurosci. 2003;107:81-4.

\section{Publisher's Note}

Springer Nature remains neutral with regard to jurisdictional claims in published maps and institutional affiliations. 